\title{
Relative Widths of Some Sets of $I_{p}^{m}$ *
}

\author{
Weiwei Xiao, Weijun Luan \\ College of Sciences, North China University of Technology, Beijing, China \\ E-mail: wwsunny@163.com
}

Received January 14, 2011; revised January 30, 2011; accepted March 10, 2011

\begin{abstract}
In this paper, the relative widths of some sets in $l_{p}^{m}$ are studied. Relative widths is the further development of Kolmogorov widths and it is a new problem in approximation theory which aroused some mathematics workers great interest recently. We present some basic propositions of relative widths and investigate relative widths of some sets (ball or ellipsoid) of $l_{p}^{m}$.
\end{abstract}

Keywords: Kolmogorov Widths, Relative Widths

\section{Introduction}

In 1984, V. N. Konovalov in [1] first proposed the definition of relative widths which is in the sense of Kolmogorov. Let $W$ and $V$ be centrally symmetric sets in a Banach space $X$. The Kolmogorov $n$-dimensional widths of $W$ relative to $V$ in $X$ (shortly, relative widths) is

$$
K_{n}(W, V, X):=\inf _{L^{n}} \sup _{f \in W} \inf _{g \in V \cap L^{n}}\|f-g\|_{X},
$$

where the infimum is taken over all $n$-dimensional subspaces $L^{n}$ of $X, n \in N$. When $V=X$ the relative widths coincides with the $n$-dimensional Kolmogorov widths (shortly, $n-K$ widths ) of $W$ in $X$, which we denote by $d_{n}(W, X)$. Of course,

$$
K_{n}(W, V, X) \geq d_{n}(W, X)
$$

for any set $V$, and if $V_{1} \subseteq V_{2}$, then

$$
K_{n}\left(W, V_{1}, X\right) \geq K_{n}\left(W, V_{2}, X\right) \text {. }
$$

Y. N. Subbotin and S. A. Telyakovskii in [7-9], V. M. Tikhomirov in [11], V. F. Babenko in [2-4], V. N. Konovalov in $[1,5,6]$, V. T. Shevaldin in [10] etc. gained many results in this field. And some Chinese mathematics workers such as Yongping Liu, Lianhong Yang in [15-17] and Weiwei Xiao in [12-14] also did some work on relative widths.

Let $l_{p}^{m}, 1 \leq p \leq \infty$, denote space of vectors

$\boldsymbol{x}=\left(x_{1}, \cdots, x_{m}\right)$ with norm

*Supported partly by National Natural Science Foundation of China (No.10471010) and partly by the project "Representation Theory and Related Topics" of the "985 Program" of Beijing Normal University and partly by Beijing Natural Science Foundation (1062004).

$$
\begin{gathered}
\|\boldsymbol{x}\|_{p}=\left(\left|x_{1}\right|^{p}+\cdots+\left|x_{m}\right|^{p}\right)^{1 / p}, 1 \leq p<\infty \\
\|\boldsymbol{x}\|_{\infty}=\max \left\{\left|x_{1}\right|, \cdots,\left|x_{m}\right|\right\}, p=\infty .
\end{gathered}
$$

Let $B_{p}:=\left\{\boldsymbol{x} \in l_{p}^{m}:\|\boldsymbol{x}\|_{p} \leq 1\right\}$ be the unit ball in $l_{p}^{m}$. Let $\boldsymbol{D}=\operatorname{diag}\left\{D_{1}, \cdots, D_{m}\right\}$ be an $m \times m$ real diagonal matrix. Without loss of generality we assume that $D_{1} \geq D_{2} \geq \cdots \geq D_{m}>0$. Let $M$ be a positive real number, set

$$
M \mathcal{D}_{p}=\left\{\boldsymbol{D} \boldsymbol{x}: \boldsymbol{x} \in R^{m},\|\boldsymbol{x}\|_{p} \leq M\right\},
$$

obviously it is ellipsoid in $l_{p}^{m}$. When $M=1$, we denote it by $\mathcal{D}_{p}$.

Theorem A: [19] For $1 \leq p \leq \infty, 1 \leq m<n$,

$$
d_{n}\left(\mathcal{D}_{p}, l_{p}^{m}\right)=D_{n+1},
$$

Similar to the proof in [18] we can get the following proposition.

Proposition 1.

1) If $W$ is a finite set of $m$ elements, then for the linear spanning subspace $\operatorname{lin}(W)$ one has

$$
K_{n}(W, \operatorname{lin}(W), X)=K_{n}(\operatorname{lin}(W), \operatorname{lin}(W), X)=0
$$

for $n \geq m$.

2) If $W_{1} \subset W$, then

$$
K_{n}\left(W_{1}, V, X\right) \leq K_{n}(W, V, X) .
$$

3) For any scalar $\alpha$, and any $W$ and $V$, one has

$$
K_{n}(\alpha W, \alpha V, X)=|\alpha| K_{n}(W, V, X) .
$$

4) $K_{0}(W, V, X) \geq K_{1}(W, V, X) \geq K_{2}(W, V, X) \geq \cdots$.

5) Let $W=K_{0}+\Gamma_{m}$, where $K_{0}$ is a bounded set and 
$\Gamma_{m}$ is a subspace of dimension $m$. If $n<m$, then $K_{n}(W, V, X)=\infty$.

6) For the convex hull $c o(W)$, if for each subspace $X_{n}$ of dimension $n, c o(W) \cap X_{n}$ is a locally sequentially compact and closed subset, then

$$
K_{n}(W, c o(W), X)=K_{n}(c o(W), c o(W), X) .
$$

7) If $Y$ is a subspace of $X$ and $W \subset Y \subseteq X$, $V \subset Y$, then

$$
K_{n}(W, V, X) \leq K_{n}(W, V, Y) .
$$

Theorem 1 For $m>n \in N, 1 \leq p \leq \infty, D_{1}>D_{n+1}$, the smallest number $M$ which makes the equalities

$$
K_{n}\left(\mathcal{D}_{p}, M \mathcal{D}_{p}, l_{p}^{m}\right)=d_{n}\left(\mathcal{D}_{p}, l_{p}^{m}\right)=D_{n+1},
$$

hold is $M_{0}:=1-\frac{D_{n+1}}{D_{1}}$, and

$$
K_{n}\left(\mathcal{D}_{p}, M \mathcal{D}_{p}, l_{p}^{m}\right)=\left\{\begin{array}{cc}
(1-M) D_{1}, & 0<M<M_{0}, \\
D_{n+1}, & M \geq M_{0} .
\end{array}\right.
$$

Theorem 2 For all $m \in N$ such that $m>1$,

$$
K_{m-1}\left(B_{1}, B_{1}, l_{\infty}^{m}\right)=\frac{1}{2} \text {. }
$$

\section{Proof of Theorems}

Proof of Theorem 1: For $\boldsymbol{x}_{0}=\left(D_{1}, 0, \cdots, 0\right)$, we have

$$
\begin{aligned}
\|\boldsymbol{x}-\boldsymbol{y}\|_{p}^{p} & =(1-M)^{p}\left(\left|x_{1}\right|^{p}+\cdots+\left|x_{n}\right|^{p}\right)+\left|x_{n+1}\right|^{p}+\cdots+\left|x_{m}\right|^{p} \\
= & (1-M)^{p}\left(\left|D_{1} z_{1}\right|^{p}+\cdots+\left|D_{n} z_{n}\right|^{p}\right)+\left|D_{n+1} z_{n+1}\right|^{p}+\cdots+\left|D_{m} z_{m}\right|^{p} \\
\leq & (1-M)^{p} D_{1}^{p}\left(\left|z_{1}\right|^{p}+\cdots+\left|z_{n}\right|^{p}\right) \\
& +(1-M)^{p} D_{1}^{p}(1-M)^{-p} D_{1}^{-p} D_{n+1}^{p}\left(\left|z_{n+1}\right|^{p}+\cdots+\left|z_{m}\right|^{p}\right) \\
\leq & (1-M)^{p} D_{1}^{p}\|z\|_{p}^{p} \leq(1-M)^{p} D_{1}^{p} .
\end{aligned}
$$

In fact, when $0<M \leq 1-\frac{D_{n+1}}{D_{1}}$, we have

$(1-M)^{-1} D_{1}^{-1} D_{n+1} \leq 1$. So we get that inequality (4).

By inequalities (2) and (4), we have that

$\forall 0<M \leq 1-\frac{D_{n+1}}{D_{1}}, \quad K_{n}\left(\mathcal{D}_{p}, M \mathcal{D}_{p}, l_{p}^{m}\right)=(1-M) D_{1}$.

From (3) and (5) we get that the smallest number $M$ which makes the equalities (1) hold is $M_{0}=1-\frac{D_{n+1}}{D_{1}}$. For $M \geq M_{0}$,

$$
K_{n}\left(\mathcal{D}_{p}, M \mathcal{D}_{p}, l_{p}^{m}\right) \leq K_{n}\left(\mathcal{D}_{p}, M_{0} \mathcal{D}_{p}, l_{p}^{m}\right)=D_{n+1} .
$$

$$
\begin{aligned}
K_{n}\left(\mathcal{D}_{p}, M \mathcal{D}_{p}, l_{p}^{m}\right) & =\inf _{L^{n} \subset l_{p}^{m}} \sup _{x \in \mathcal{D}_{p}} \inf _{y \in M \mathcal{D}_{p} \cap L^{n}}\|\boldsymbol{x}-\boldsymbol{y}\|_{p} \\
& \geq \sup _{x \in \mathcal{D}_{p}} \inf _{y \in M \mathcal{D}_{p}}\|\boldsymbol{x}-\boldsymbol{y}\|_{p} \\
& \geq \inf _{y \in M \mathcal{D}_{p}}\left\|\boldsymbol{x}_{0}-\boldsymbol{y}\right\|_{p} \\
& =D_{1}(1-M) .
\end{aligned}
$$

That is

$$
K_{n}\left(\mathcal{D}_{p}, M \mathcal{D}_{p}, l_{p}^{m}\right) \geq D_{1}(1-M), \quad \forall 0<M \leq 1 .
$$

In order to make the equalities (1) hold, we have that

$$
D_{n+1} \geq D_{1}(1-M) \text {, }
$$

that is $M \geq 1-\frac{D_{n+1}}{D_{1}}$.

For $0<M \leq 1-\frac{D_{n+1}}{D_{1}}$, we will prove that

$$
K_{n}\left(\mathcal{D}_{p}, M \mathcal{D}_{p}, l_{p}^{m}\right) \leq(1-M) D_{1} \text {. }
$$

For each $\boldsymbol{x}=\boldsymbol{D z} \in \mathcal{D}_{p},\|\mathbf{z}\|_{p} \leq 1$, set $y=\left(M x_{1}, \cdots, M x_{n}, 0, \cdots, 0\right) \in L^{n} \cap M \mathcal{D}_{p}$. When $p=\infty$, the inequality (4) is trivial, so we only need to prove the case of $1 \leq p<\infty$. 
By proposition (6) we know that

$$
K_{m-1}\left(B_{1}, B_{1}, l_{\infty}^{m}\right)=K_{m-1}\left(W, B_{1}, l_{\infty}^{m}\right),
$$

where

$$
W=\left\{\left(0, \cdots, 0,( \pm 1)_{i}, 0, \cdots, 0\right): i=1, \cdots, m, i \text { represent the } i \text { th coordinate }\right\}
$$

Set

$$
L_{*}^{m-1}:=\left\{\boldsymbol{x} \in R^{m}: x_{1}+x_{2}+\cdots+x_{m}=0\right\} .
$$

For $a=\left(0, \cdots, 0,( \pm 1)_{i}, 0, \cdots, 0\right) \in W$, set

$$
b=\left(0, \cdots, 0,( \pm 1 / 2)_{i},(\mp 1 / 2)_{i+1}, 0, \cdots, 0\right) \in L_{*}^{m-1} \cap B_{1},
$$

$i=1, \cdots, d$, when $i=d, i+1$ represent the 1 st coordinate, we get $\|a-b\|_{\infty}=1 / 2$. So we proved

$$
K_{m-1}\left(W, B_{1}, l_{\infty}^{m}\right) \leq 1 / 2 \text {, }
$$

which means that inequality (9) is valid. The proof of Theorem 2 is complete.

\section{References}

[1] V. N. Konovalov, "Estimates of Diameters of Kolmogorov Type for Classes of Differentiable Periodic Functions,” Matematicheskie Zametki, Vol. 35, No. 3, 1984, pp. 369-380.

[2] V. F. Babenko, "On the Relative Widths of Classes of Functions with Bounded Mixed Derivative,” East Journal on Approximations, Vol. 2, No. 3, 1996, pp. 319-330.

[3] V. F. Babenko, “Approximation in the Mean under Restriction on the Derivatives of the Approximating Functions, Questions in Analysis and Approximations,” Institute of the Mathematics of the Ukrainean Academy of Science, Kiev, 1989, pp. 9-18. (in Russian)

[4] V. F. Babenko, “On Best Uniform Approximations by Splines in the Presence of Restrictions on Their Derivatives," Mathematical Notes, Vol. 50, No. 6, 1991, pp. 1227-1232. Translated from Matematicheskie Zametki, Vol. 50, No. 6, 1991, pp. 24-30.

[5] V. N. Konovalov, "Approximation of Sobolev Classes by Their Sections of Finite Dimensional,” Ukrainian Mathematical Journal, Vol. 54, No. 5, 2002, pp. 795-805. doi:10.1023/A:1021635530578

[6] V. N. Konovalov, "Approximation of Sobolev Classes by Their Finite-Dimensional Sections,” Matematicheskie Zametki, Vol. 72, No. 3, 2002, pp. 370-382.

[7] Y. N. Subbotin and S. A. Telyakovskii, "Exact Values of Relative Widths of Classes of Differentiable Functions," Mathematical Notes, Vol. 65, No. 6, 1999, pp. 731-738. Translated from Matematicheskie Zametki, Vol. 65, No. 6, 1999, pp. 871-879.

[8] Y. N. Subbotin and S. A. Telyakovskii, "Relative Widths of Classes of Differentiable Functions in the $L^{2}$ Metric,” Russian Mathematical Surveys, Vol. 56, No. 4, 2001, pp. 159-160.

[9] Y. N. Subbotin and S. A. Telyakovskii, "On Relative Widths of Classes of Differentiable Functions,” Proceedings of the Steklov Institute of Mathematics, 2005, pp. 243-254.

[10] V. T. Shevaldin, "Approximation by Local Trigonometric Splines,” Mathematical Notes, Vol. 77, No. 3, 2005, pp. 326-334.

[11] V. M. Tikhomirov, "Some Remarks on Relative Diameters,” Banach Center Publications, Vol. 22, 1989, pp. 471-474.

[12] W. W. Xiao, "Relative Widths of Classes of Functions Defined by a Self-Conjugate Linear Differential Operator in $L_{q}(T)$ (in Chinese)," Chinese annals of Mathematics, Vol. 29A, No. 5, 2008, pp. 679-688.

[13] W. W. Xiao, "Relative Infinite-Dimensional Width of Sobolev Classes," Journal of Mathematical Analysis and Applications, Vol. 369, No. 2, 2010, pp. 575-582. doi:10.1016/j.jmaa.2010.03.050

[14] Y. P. Liu and W. W. Xiao, "Relative Average Widths of Sobolev Spaces in $L_{2}\left(\mathrm{R}^{d}\right)$," Analysis Mathematica, Vol. 34, No. 1, 2008, pp. 71-82. doi:10.1007/s10476-008-0107-8

[15] Y. P. Liu and L. H. Yang, "Relative Width of Smooth Classes of Multivariate Periodic Functions with Restrictions on Interated Laplace Derivatives in The $L_{2}$-MetRic,” Acta Mathematica Scientia, Vol. 26B, No. 4, 2006, pp. 720-728. doi:10.1016/S0252-9602(06)60098-2

[16] Y. P. Liu and L. H. Yang, "Relative Widths of Smooth Factions Determined by Fractional Order Derivatives," Journal of complexity, Vol. 24, No. 2, 2008, pp. 259-282.

[17] L. H. Yang and Y. P. Liu, "Relative Widths of Smooth Functions Determined by Linear Differential Operator," Journal of Mathematical Analysis and Applications, Vol. 351, No. 2, 2009, pp. 734-746. doi:10.1016/j.jmaa.2008.11.009

[18] G. G. Lorentz, M. V. Golitschek, and Y. Makovoz, "Constructive Approximation,” Springer, Berlin, 1996.

[19] A. Pinkus, "N-Widths in Approximation Theory," Springer, Berlin, 1985. 